Original Research Paper

\title{
Genotype Screening of Bovine Brachyspina in Taiwan Holstein Cows
}

\author{
Chun Hsuan Chao, Yi Ming Chen and Kuo Hua Lee \\ Hsinchu Branch, Livestock Research Institute, Council of Agriculture, \\ Executive Yuan, No. 207-5, Bitoumian, Wuhoo Village, Sihoo Township, Miaoli County, Taiwan
}

\author{
Article history \\ Received: 19-05-2020 \\ Revised: 11-07-2020 \\ Accepted: 25-07-2020 \\ Corresponding Author: \\ Chun Hsuan Chao \\ Hsinchu Branch, Livestock \\ Research Institute, Council of \\ Agriculture, Executive Yuan, \\ No. 207-5, Bitoumian, Wuhoo \\ Village, Sihoo Township, \\ Miaoli County, Taiwan \\ Email: jschao@tlri.gov.tw
}

\begin{abstract}
This study analyzed carrier frequencies of the genetic defect bovine brachyspina (BS) in the Holstein cows in Taiwan. In total, 1688 cows from 31 herds were included in the analysis, performed using the Igenity-Prime 50K single-nucleotide polymorphism chip. Of the 1688 cows, $68(5.09 \%)$ were BS carriers (BY), a frequency lower than that reported in Taiwan previously. In Taiwan, the import of frozen BY bull semen has been discontinued since the implementation of an import censorship act in 2015. Pedigree analysis results indicated that $70 \%$ of the defective genotype was inherited from dam carriers. Through mating programs, the defect allele has remained in Taiwan Holstein herds. Regular screening for genetic defects is necessary eliminate the recessive BS gene from the Taiwan Holstein population.
\end{abstract}

Keywords: Bovine Brachyspina, Holstein Cows, Genetic Defect

\section{Introduction}

Bovine brachyspina (BS) was first identified in Denmark in 2006 (Agerholm et al., 2006), subsequently, it was found in the Netherlands and Denmark (Agerholm and Peperkamp, 2007; Charlier et al., 2012), Italy (Testoni et al., 2008), Canada (Agerholm et al., 2010), Germany (Buck et al., 2010) and the United States (VanRaden et al., 2011). BS is a recessive lethal genetic disorder caused by a $3.3-\mathrm{kb}$ fragment deletion in the FANCI gene on the bovine chromosome 21 (Charlier et al., 2012) and it can lead to early abortion or stillbirth in pregnant cows and thus cause economic loss. The abbreviation BY indicates that cattle are heterozygous for the BS allele, whereas TY indicates that cattle are homozygous for the allele (i.e., healthy). It was traced back to the common ancestors, Sweet Haven Tradition (NAAB: 023HO00206) (Testoni et al., 2008; Charlier et al., 2012). Through their high milk production descent bulls to spread the BS defect allele. The BS allele was introduced into the Taiwan Holstein cattle population through import of frozen bull semen. Effective screening to prevent further transmission of the BS allele is necessary. For simultaneous genetic screening of defective alleles in Holstein cows, Zhang et al. (2020) developed the Kompetitive Allele-Specific Polymerase chain reaction (KASP) assay for discrimination of wild- type alleles from eight defective alleles associated with Holstein cows. However, the KASP assay is only appropriate for analysis of small to moderate numbers of loci. Multiplexed, chip-based, high-throughput genotyping technology is preferred for analysis of large numbers of genomic Single-Nucleotide Polymorphisms (SNPs) (Zhang et al., 2020). The Igenity-Prime $50 \mathrm{k}$ SNP chip, which comprises nearly 42,000 genetic markers, is cost-effective, provides a comprehensive genomic profile and yields highly accurate results. The results included more than 45 traits approved by the Council of Dairy Cattle Breeding (CDCB) in the United States and included content for coat color, milk components and genetic conditions.

Liaw et al. (2015; 2017) and Lai et al. (2017) have investigated BS carrier prevalence in Taiwan. Nevertheless, the current study evaluated whether the BS allele frequency displays a reduction rate and to determine its transmission route.

\section{Materials and Methods}

In total, 1688 cows were randomly selected from 31 herds $(9,10,11$ and 1 from Northern, Central, Southern and Eastern Taiwan, respectively) between 2017 and 2019. Neogen's patented hair and blood sample collection cards were used to hold hair follicles and 
collect blood for DNA processing and archiving. Approximately 30 hair roots were pulled from each cow. A syringe was used to take blood samples from the vein on the underside of the tail. Two to three drops of blood were allowed to drip onto the collection portion of the sample collection cards. The sample collection cards were then processed at $75^{\circ} \mathrm{C}$ for $30 \mathrm{~min}$. The animal use protocol was reviewed and approved by the Institutional Animal Care and Use Committee of the Taiwan Livestock Research Institute (LRI-IACUC107-5, LRIIACUC108-1). The documents were accompanied by the sampled cows' information, including their birthdate, sire, dam and granddam. The sample collection cards were mailed to Neogen Corporation (NE), where the samples were tested using Igenity-Prime 50k SNP chips. The CDCB-certified laboratory at Neogen uses the Illumina Infinium XT genotyping assay platform (Illumina, 2017), with the following recommended workflow: "On day one, DNA is amplified, enzymatically fragmented, precipitated and resuspended. Samples are then hybridized to BeadChips during an overnight incubation, where DNA anneals to locusspecific 50-mer probes covalently linked to one of the Infinium bead types. On day two, the Infinium XT workflow continues with enzymatic base extension to confer allelic specificity, followed by fluorescent staining. The iScan System detects the fluorescence intensities of the beads and Illumina software automatically performs analysis and genotype calling." The genotype data were emailed to the CDCB for genetic evaluation. The genetic evaluation results included key traits, production traits, reproductive traits, type traits, genetic defects and parental confirmation.

\section{Results and Discussion}

The BS genotype frequencies of the sampled cows are shown in Table 1. BY carriers were found in 17 of 31 herds, with 1-25 cows in each herd, with carrier frequencies of $1.85-15 \%$. The total carrier frequency of $5.09 \%$ was lower than that reported in the United States (6\%) (VanRaden et al., 2011), the Netherlands (7.4\%) (Charlier et al., 2012) and Poland (10.26\%) (Ruść and Kaminski, 2015) and higher than that reported in China (2.6\%) (Zhang et al., 2020). It was also lower than that reported by previous Taiwanese studies, in which 409, 172 and 436 cattle from 4, 4 and 9 herds, respectively, with BY carrier frequencies of $8.56,12.21$ and $6.65 \%$, respectively, were sampled (Liaw et al., 2015; 2017; Lai et al., 2017). The variations in carrier prevalence were consistent with those found in a study in Turkey and were probably attributable to differences between studies in sample size, farms and regions (Avanus and Altınel, 2017).
Table 1: BS carrier frequencies among 31 herds of Holstein cows in Taiwan

\begin{tabular}{|c|c|c|c|c|}
\hline \multirow[b]{2}{*}{ Herd } & \multirow{2}{*}{$\begin{array}{l}\text { No. of } \\
\text { genotyped cattle }\end{array}$} & \multicolumn{2}{|c|}{ BS genotype } & \multirow{2}{*}{$\begin{array}{l}\text { Frequency of } \\
\text { carrier (\%) }\end{array}$} \\
\hline & & Normal & Carrier & \\
\hline 1 & 30 & 28 & 2 & 6.67 \\
\hline 2 & 109 & 104 & 5 & 4.59 \\
\hline 3 & 76 & 76 & 0 & 0.00 \\
\hline 4 & 163 & 157 & 6 & 3.68 \\
\hline 5 & 20 & 20 & 0 & 0.00 \\
\hline 6 & 19 & 18 & 1 & 5.26 \\
\hline 7 & 67 & 61 & 6 & 8.96 \\
\hline 8 & 20 & 18 & 2 & 10.00 \\
\hline 9 & 39 & 34 & 5 & 12.82 \\
\hline 10 & 40 & 34 & 6 & 15.00 \\
\hline 11 & 10 & 10 & 0 & 0.00 \\
\hline 12 & 10 & 10 & 0 & 0.00 \\
\hline 13 & 9 & 9 & 0 & 0.00 \\
\hline 14 & 9 & 8 & 1 & 11.11 \\
\hline 15 & 115 & 109 & 6 & 5.22 \\
\hline 16 & 10 & 10 & 0 & 0.00 \\
\hline 17 & 43 & 42 & 1 & 2.33 \\
\hline 18 & 5 & 5 & 0 & 0.00 \\
\hline 19 & 20 & 20 & 0 & 0.00 \\
\hline 20 & 11 & 11 & 0 & 0.00 \\
\hline 21 & 409 & 384 & 25 & 6.11 \\
\hline 22 & 23 & 21 & 2 & 8.70 \\
\hline 23 & 19 & 18 & 1 & 5.26 \\
\hline 24 & 10 & 10 & 0 & 0.00 \\
\hline 25 & 242 & 228 & 14 & 5.79 \\
\hline 26 & 6 & 6 & 0 & 0.00 \\
\hline 27 & 10 & 9 & 1 & 10.00 \\
\hline 28 & 9 & 9 & 0 & 0.00 \\
\hline 29 & 10 & 10 & 0 & 0.00 \\
\hline 30 & 17 & 17 & 0 & 0.00 \\
\hline 31 & 108 & 106 & 2 & 1.85 \\
\hline Total & 1688 & 1602 & 86 & 5.09 \\
\hline
\end{tabular}

\section{HOUSA62769059}
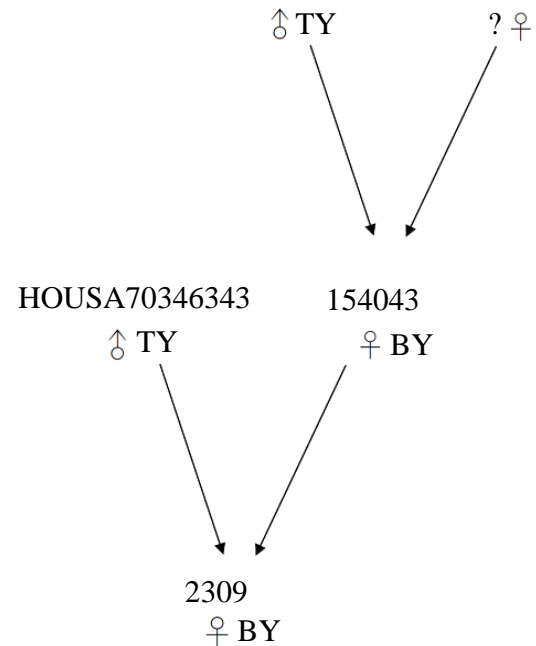

Fig. 1: Pedigree analysis of BS genotype transmission. TY, BS-free animals; BY, BS carriers 


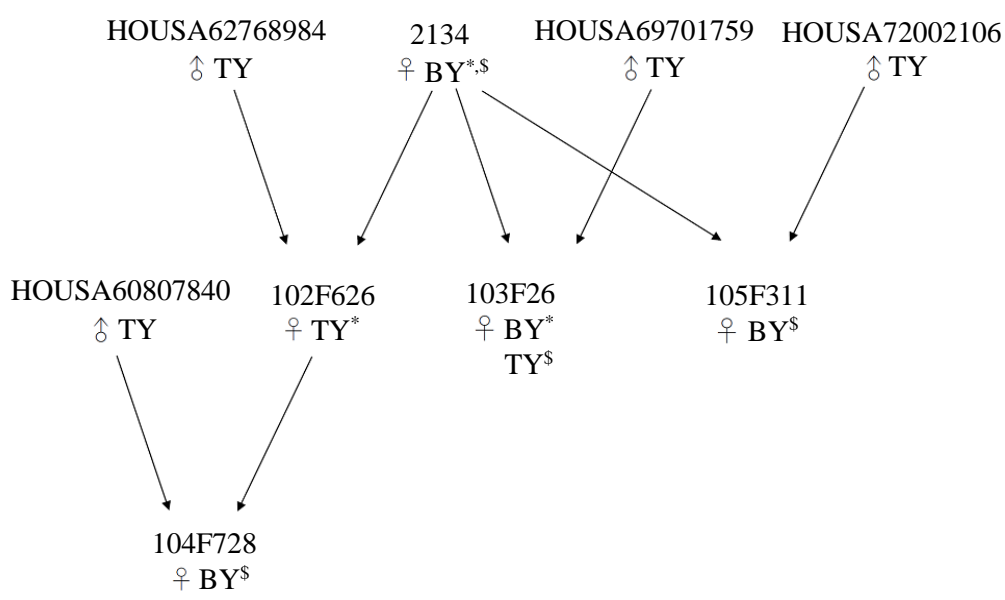

Fig. 2: Differences between BY and TY genotypes among Taiwan Holstein cows between a 2017 study by Lai et al. and the present study. *indicates genotypes analyzed in a past study (Lai et al., 2017). ${ }^{\$}$ indicates the genotypes analyzed in the present study. TY, BS-free animals; BY, BS carriers.

Table 2: The statistical numbers of genotype of the sire of BS carrier and the percentage of inherited BS frequency from maternal among the 31 herds

\begin{tabular}{|c|c|c|c|c|c|}
\hline \multirow[b]{2}{*}{ Herd } & \multirow[b]{2}{*}{ BS carrier } & \multicolumn{3}{|c|}{ BS genotype of sire of BS carrier } & \multirow{2}{*}{$\begin{array}{l}\text { Inherited BS frequency } \\
\text { from maternal }(\%)\end{array}$} \\
\hline & & Normal & Carrier $^{@}$ & Uncertainty & \\
\hline 1 & 2 & 2 & 0 & 0 & 100.00 \\
\hline 2 & 5 & 5 & 0 & 0 & 100.00 \\
\hline 4 & 6 & 5 & 0 & $1^{\#}$ & 100.00 \\
\hline 6 & 1 & 1 & 0 & 0 & 100.00 \\
\hline 7 & 6 & 3 & $\begin{array}{l}3 \\
\text { HOCAN000007588018 (3) }\end{array}$ & 0 & 50.00 \\
\hline 8 & 2 & 1 & 0 & $1^{\$}$ & 100.00 \\
\hline 9 & 5 & 4 & $\begin{array}{l}1 \\
\text { HOUSA000060839913 (1) }\end{array}$ & 0 & 80.00 \\
\hline 10 & 6 & 1 & $\begin{array}{l}5 \\
\text { HOCAN000007588022 (4) } \\
\text { HOCAN000007588018 (1) }\end{array}$ & 0 & 16.67 \\
\hline 14 & 1 & 1 & 0 & 0 & 100.00 \\
\hline 15 & 6 & 3 & $\begin{array}{l}2 \\
\text { HOCAN000007588022 (2) }\end{array}$ & $1^{\$}$ & 60.00 \\
\hline 17 & 1 & 1 & 0 & 0 & 100.00 \\
\hline 21 & 25 & 11 & $\begin{array}{l}13 \\
\text { HOUSA000052357952 (1) } \\
\text { HOUSA000060839913(1) } \\
\text { HOUSA000069990180(11) }\end{array}$ & $1^{\$}$ & 45.83 \\
\hline 22 & 2 & 2 & 0 & 0 & 100.00 \\
\hline 23 & 1 & 1 & 0 & 0 & 100.00 \\
\hline 25 & 14 & 14 & 0 & 0 & 100.00 \\
\hline 27 & 1 & 1 & 0 & 0 & 100.00 \\
\hline 31 & 2 & 1 & $\begin{array}{l}1 \\
\text { HOUSA000069990180 (1) }\end{array}$ & 0 & 50.00 \\
\hline Total & 86 & 57 & 25 & 4 & 69.51 \\
\hline
\end{tabular}

${ }^{\circledR}$ all BS carriers traced back to Sweet Haven Tradition.

\$Unknown sire.

\#Sire without BS genotype information.

The pedigree analysis results demonstrated that the sire of all $25 \mathrm{BY}$ cows (Table 2) could be traced back to an ancestor carrier, the US Holstein sire Sweet Haven
Tradition (NAAB: 023HO00206). Without counting the cows with unknown sires or sires without BS genotype information, $69.51 \%$ (57 of 82 ) of sires of the BY cows 
had the normal TY genotype. Therefore, almost $70 \%$ of the BY genotype was inherited from the dams, which also had the BY genotype. Moreover, in 11 of the 31 herds, $100 \%$ of the BY genotype was inherited from the dams. The results of the pedigree analysis for $\mathrm{BS}$ transmission demonstrated that the BS allele could be transmitted across three generations even if the sires had the normal (TY) genotype (Fig. 1). Such cases of recessive transmission through dams have also been found in Poland (Ruść et al., 2013).

Some of the analyzed results were different from those in a past study. For example, Cattle 103F26 was previously determined to have the BY genotype (Lai et al., 2017), but in the present study, it was found to have the TY genotype. In a contrasting case, Cattle 102F626 was previously determined to have the TY genotype. Although she died before sampling, her female calf, Cattle 104F728, was demonstrated the BY genotype in the present study. Because HOUSA60807840, the sire of Cattle 104F728, had the TY genotype, the defective allele carried by Cattle 104F728 was theoretically inherited maternally from Cattle 102F626. Moreover, Cattle 2134, the granddam of Cattle 104F728, also had the BY genotype. Therefore, it can be inferred that Cattle 102F626 should have the BY genotype, which contradicts what Lai et al. (2017) found. The polymerase chain reaction detection method was probably not inaccurate; rather, the lack of data editing correction may have led to reverse genotyping between Cattle 103F26 and Cattle 102F626 (Fig. 2). The BeadArray SNP genotyping platform (Illumina, CA) used in this study has a high throughput and uses a popular allele discrimination method to generate allelespecific products (Kim and Misra, 2007; Oliphant et al., 2002). This process is accurate, rapid and cost-effective. Moreover, the commercial genomic evaluation service provided by CDCB-certified laboratories can be used as a screening model for many countries that do not regularly monitor autosomal recessive disorders or have not yet conducted surveys of such disorders.

Because Taiwan often imports cattle and frozen bull semen, the introduction of genetic defects to Taiwan's dairy herds has been considerably affected by bulls worldwide (Agerholm et al., 2010; Charlier et al., 2012). Routine screening for genetic defects and implementation of the Imported Frozen Bull Semen Censorship Act can effectively prevent inferior cattle breeds from entering Taiwan. The BS allele no longer enters Taiwan Holstein cattle population because frozen BY bull semen has not been imported since 2015. Therefore, the BY genotype has mostly been inherited from carrier dams. Although the carrier frequency is less than $6 \%$ and decreases annually, close monitoring is essential to prevent the BS allele from remaining in the herds. In addition, because the BS carrier frequency remains relatively high in some cattle herds, dairy farmers should pay more attention to mating programs.

\section{Conclusion}

Of the 1688 included cows, 68 (5.09\%) had the BY phenotype; this frequency is lower than that noted in previous studies in Taiwan. Pedigree analysis revealed that approximately $70 \%$ of the BS genotype was maternally inherited. Genomic evaluation by CDCB-certified laboratories can be used as a screening model to closely monitor the BS defect in Taiwan Holstein cattle herds.

\section{Acknowledgment}

This work was supported by the Council of Agriculture under grant No. 108AS-2.1.2-L1(1). This manuscript was edited by Wallace Academic Editing.

\section{Author's Contributions}

Chun Hsuan Chao and Kuo Hua Lee: Research Conceptualization and Design.

Chun Hsuan Chao and Yi Ming Chen: Data

Collection and Interpretation.

Chun Hsuan Chao: Writing and Editing.

\section{References}

Agerholm, J. S., McEvoy, F., \& Arnbjerg, J. (2006). Brachyspina syndrome in a Holstein calf. Journal of Veterinary Diagnostic Investigation, 18(4), 418-422.

Agerholm, J. S., DeLay, J., Hicks, B., \& Fredholm, M. (2010). First confirmed case of the bovine brachyspina syndrome in Canada. The Canadian Veterinary Journal, 51(12), 1349.

Agerholm, J. S., \& Peperkamp, K. (2007). Familial occurrence of Danish and Dutch cases of the bovine brachyspina syndrome. BMC Veterinary research, 3(1), 8.

Avanus, K., \& Altınel, A. (2017). Inherited diseases of Holstein cattle: Story so far in Turkey. Journal of Istanbul Veterinary Sciences, 1(2), 40-46.

Buck, B. C., Ulrich, R., Woehlke, A., Kuiper, H., Baumgaertner, W., \& Distl, O. (2010). Vertebral and multiple organ malformations in a black and white German Holstein calf. Berliner und Munchener Tierarztliche Wochenschrift, 123(5-6), 251-255.

Charlier, C., Agerholm, J. S., Coppieters, W., KarlskovMortensen, P., Li, W., De Jong, G., ... \& Ahariz, N. (2012). A deletion in the bovine FANCI gene compromises fertility by causing fetal death and brachyspina. PloS one, 7(8), e43085. 
Illumina. (2017). Infinium XT Assay. Document \# 1000000016440v01.

Kim, S., \& Misra, A. (2007). SNP genotyping: technologies and biomedical applications. Annual Review of Biomedical Engineering, 9, 289-320.

Lai, F. Y., Chen, Y. H., Tu, P. A., Shiau, J. W., \& Shiu, J. S. (2017). Feasibility and frequency of gene detection of bovine brachyspina syndrome of Holstein cows by real-time polymerase chain reaction. Journal of the Chinese Society of Animal Science, 46, 13-30.

Liaw, R.B., Chen, J. C., Chen, W. C., Tsay, S. S., \& Huang, S. L. (2015). The pilot study on genotype screening of brachyspina syndrome on dairy cattle herds in Taiwan. Taiwan Livestock Res, 48, 210-215.

Liaw, R. B., Chen, J. C., Wu, M. C., \& Huang, S. L. (2017). Development of a new method for rapid genotyping brachyspina syndrome in dairy cattle. Taiwan Livestock Res, 50, 213-218.

Oliphant, A., Barker, D. L., Stuelpnagel, J. R., \& Chee, M. S. (2002). BeadArray ${ }^{\mathrm{TM}}$ technology: enabling an accurate, cost-effective approach to high-throughput genotyping. Biotechniques, 32(sup), S56-S61.
Ruść, A., Hering, D., Puckowska, P., Barcewicz, M., \& Kaminski, S. (2013). Screening of Polish HolsteinFriesian bulls towards eradication of complex vertebral malformation (CVM) carriers. Polish Journal of Veterinary Sciences, 16(3), 579-581.

Ruść, A., \& Kaminski, S. (2015). Detection of brachyspina carriers within Polish Holstein-Friesian bulls. Polish Journal of Veterinary Sciences, 18(2), 453-454.

Testoni, S., Diana, A., Olzi, E., \& Gentile, A. (2008). Brachyspina syndrome in two Holstein calves. The Veterinary Journal, 177(1), 144-146.

VanRaden, P. M., Olson, K. M., Null, D. J., \& Hutchison, J. L. (2011). Harmful recessive effects on fertility detected by absence of homozygous haplotypes. Journal of Dairy Science, 94(12), 6153-6161.

Zhang, Y., Liang, D., Huang, H., Yang, Z., Wang, Y., Yu, Y., ... \& Xiao, W. (2020). Development and application of KASP assays for rapid screening of 8 genetic defects in Holstein cattle. Journal of Dairy Science, 103(1), 619-624. 